\title{
Approximate Bayesian Computation with composite score functions
}

\author{
Erlis Ruli*, Nicola Sartori and Laura Ventura \\ Department of Statistical Sciences, University of Padova, Italy \\ *ruli@stat.unipd.it, sartori@stat.unipd.it, ventura@stat.unipd.it
}

August 27, 2018

\begin{abstract}
Both Approximate Bayesian Computation (ABC) and composite likelihood methods are useful for Bayesian and frequentist inference, respectively, when the likelihood function is intractable. We propose to use composite likelihood score functions as summary statistics in $\mathrm{ABC}$ in order to obtain accurate approximations to the posterior distribution. This is motivated by the use of the score function of the full likelihood, and extended to general unbiased estimating functions in complex models. Moreover, we show that if the composite score is suitably standardised, the resulting ABC procedure is invariant to reparameterisations and automatically adjusts the curvature of the composite likelihood, and of the corresponding posterior distribution. The method is illustrated through examples with simulated data, and an application to modelling of spatial extreme rainfall data is discussed.
\end{abstract}

Keywords: Complex model; Composite marginal likelihood; Likelihood-free inference; Pairwise likelihood; Tangent exponential model; Unbiased estimating function. 


\section{Introduction}

The summary of the data on a given model offered by the likelihood function is the key ingredient of all likelihood-based inferential methods. However, likelihood inference, both frequentist and Bayesian, is difficult or even impossible to perform when the likelihood is analytically or computationally intractable. This usually occurs in the presence of complex models, such as models with complicated dependence structures or in models with many latent variables.

In these situations, for frequentist or Bayesian inference, surrogates of the ordinary likelihood are useful. A notable contribution is given by composite likelihoods (Lindsay, 1988), which are based on the composition of suitable lower dimensional densities, such as bivariate marginal (Cox \& Reid, 2004), conditional or full conditional densities (Varin et al., 2011). The use of composite likelihoods has been widely advocated in different complex applications of frequentist inference (see Varin et al., 2011, for a general review, and Larribe \& Fearnhead, 2011, for a review in genetics).

From a Bayesian perspective, when the computation of the likelihood is impracticable, but it is easy to simulate from the model, an approximation of the posterior distribution can be obtained by Approximate Bayesian Computation (ABC). The idea of $\mathrm{ABC}$ is to simulate from the model for different parameter values, and to keep those values that produce simulated datasets that approximately match the observed data (see Beaumont, 2010; Marin et al., 2012). The most popular ABC approach is to consider an approximate matching of some summary statistics, evaluated at the observed and simulated data, by means of suitable distances. When the statistics are sufficient for the parameters of the model, this method leads to the exact posterior distribution as the distance tends to zero. However, in realistic applications sufficient statistics are not available and the practitioner must resort to a careful selection of data summaries.

In this paper we propose the use of a scaled composite likelihood score function as summary statistic in ABC. The idea is motivated by the use of the score function when the full likelihood is available and is then extended to composite likelihood score functions in complex models. The ABC algorithm based on composite score functions (ABC-cs) searches for 
parameter values of the model of interest that produce simulated data which lead to composite score values - at the observed maximum composite likelihood estimate - close to those based on the original data. The composite score statistic is rescaled with the corresponding information in order to take into account the amount of information on different parameter components. This rescaling has also the effect of making the ABC-cs procedure invariant to reparameterisations.

Although ABC-cs is not universally applicable, when it can be employed, e.g. when sensible composite likelihoods can be defined for the given model, it has several useful features. First of all, the summary statistic has dimension equal to the number of parameters, and it inherits, by construction, structural stochastic characteristics of the model. Such statistic is also generally easy to compute, being often available analytically. Moreover, ABC-cs automatically adjusts the curvature of the composite likelihood and of the corresponding posterior distribution. Indeed, composite likelihoods typically do not satisfy the second Bartlett identity, also known as the information identity, and this usually leads to overly concentrated posterior distributions (Pauli et al., 2011; Smith \& Stephenson, 2009). Hence, the straightforward use of the composite likelihood as a replacement to the full likelihood in Bayes' formula does not generally give a valid posterior distribution. For this reason, Pauli et al. (2011) propose to first rescale the composite likelihood at the mode and then use this calibrated version in Bayes' theorem. This certainly improves inference, but sometimes may lead to the opposite problem of overestimating the variability in the posterior. From this point of view, at least in the examples considered here, the ABC-cs method gives better results, although computationally it may be more demanding, as is often the case with $\mathrm{ABC}$ methods.

There have been other attempts to merge composite likelihoods with the ABC framework. For instance, Mengersen et al. (2013) use the composite score function with the empirical likelihood to produce an approximate and weighted posterior sample. Their approach is not $\mathrm{ABC}$ in the usual common sense, as it does not simulate from the full model. Also Barthelmé \& Chopin (2014, Sec. 7.1) mention the use of composite likelihoods in order to reduce the computational complexity of $\mathrm{ABC}$, but they do not use the composite score as summary statistic. 
Our approach is similar in spirit to indirect inference (Gourieroux et al., 1993; Heggland \& Frigessi, 2004), as also the ABC-cs method relies on an auxiliary model likelihood, that is the composite likelihood. However, ABC-cs is less computationally demanding since it does not require repeated maximisation for each simulated dataset. The indirect inference method within ABC has been discussed by Drovandi et al. (2011). More recently, also Gleim \& Pigorsch (2013) and Drovandi et al. (2014) advocate the use of score functions based on auxiliary models as ABC summary statistics.

In Section 2 some background on $\mathrm{ABC}$ and composite likelihood methods is given. The proposed ABC-cs algorithm is presented in Section 3. Section 4 illustrates the method by two examples, while Section 5 gives an application to spatial extreme data. Section 6 concludes the paper.

\section{Statistical methods}

\subsection{ABC algorithms}

Let $\pi(\theta)$ be a prior distribution for the parameter $\theta \in \Theta \subseteq \mathbb{R}^{d}, L(\theta)=L(\theta ; y)=f(y ; \theta)$ the likelihood function based on data $y$ and $\pi(\theta \mid y) \propto \pi(\theta) L(\theta)$ the posterior distribution of $\theta$. Suppose that $L(\theta)$ is unavailable for mathematical or computational reasons.

The primary purpose of $\mathrm{ABC}$ algorithms is to approximate the posterior distribution when other methods, such as Markov chain Monte Carlo (MCMC), data augmentation, importance sampling or Laplace approximation cannot be used, but when the data from $f(y ; \theta)$ can be easily simulated. Let $\eta(\cdot)$ be a set of summary statistics, $\rho(\cdot, \cdot)$ a distance function and $\epsilon>0$ a tolerance threshold. Moreover, let $y^{\text {obs }}$ be the observed data. Then the ABC accept-reject algorithm (Algorithm 1) 


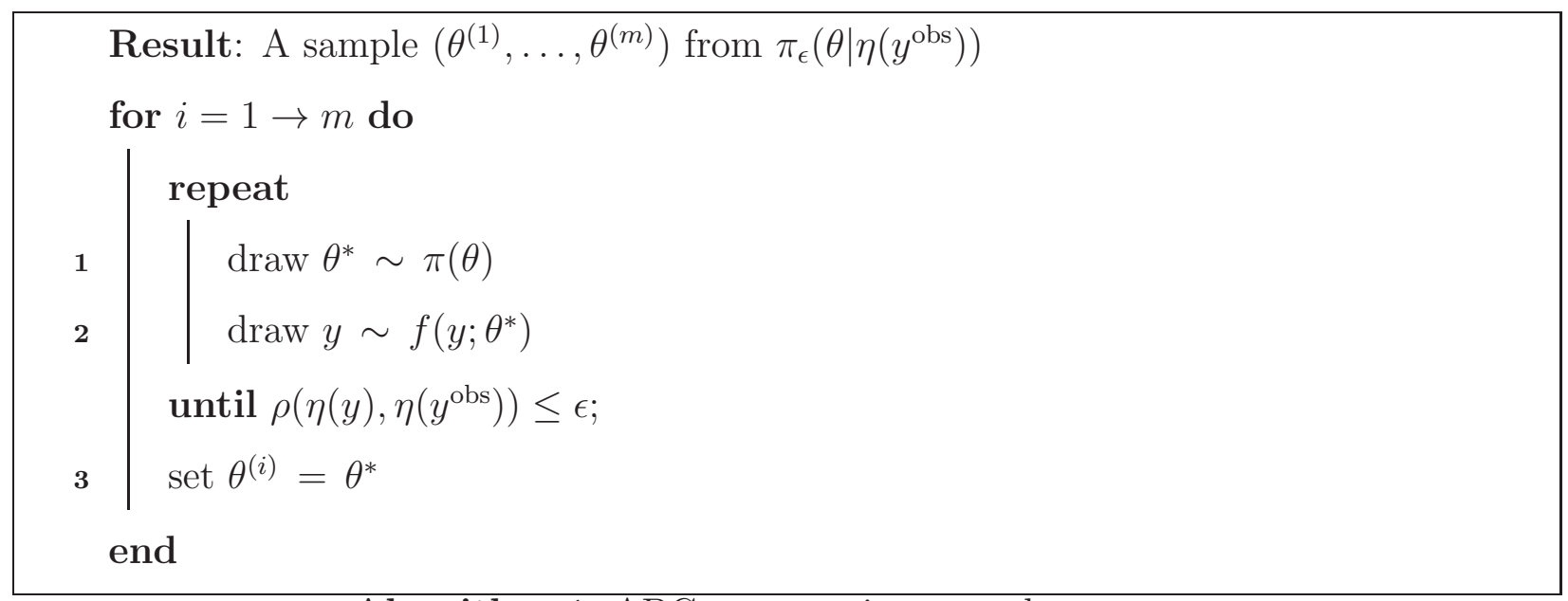

Algorithm 1: ABC accept-reject sampler.

samples from the joint distribution

$$
\pi_{\epsilon}\left(\theta, y \mid \eta\left(y^{\mathrm{obs}}\right)\right)=\frac{\pi(\theta) f(y ; \theta) \mathbb{I}_{A_{\epsilon, y} \mathrm{obs}}(y)}{\int_{A_{\epsilon, y} \mathrm{obs} \times \Theta} \pi(\theta) f(y ; \theta) d y d \theta},
$$

where $\mathbb{I}_{A_{\epsilon, y \text { obs }}}(y)$ is the indicator function of the set $A_{\epsilon, y^{\text {obs }}}(y)=\left\{y: \rho\left(\eta(y), \eta\left(y^{\mathrm{obs}}\right)\right) \leq \epsilon\right\}$, and it produces an approximation to the posterior distribution $\pi\left(\theta \mid y^{\text {obs }}\right)$, given by

$$
\pi_{\epsilon}\left(\theta \mid \eta\left(y^{\mathrm{obs}}\right)\right)=\int \pi_{\epsilon}\left(\theta, y \mid \eta\left(y^{\mathrm{obs}}\right)\right) d y .
$$

If $\epsilon \rightarrow 0$, then $\pi_{\epsilon}\left(\theta \mid \eta\left(y^{\text {obs }}\right)\right) \rightarrow \pi\left(\theta \mid \eta\left(y^{\text {obs }}\right)\right)$ (Blum, 2010). In addition, if $\eta(\cdot)$ is sufficient, then $\pi_{\epsilon}\left(\theta \mid \eta\left(y^{\mathrm{obs}}\right)\right) \rightarrow \pi\left(\theta \mid y^{\mathrm{obs}}\right)$ (see, for instance, Marin et al., 2012).

The threshold $\epsilon$ cannot be fixed to zero, for computational efficiency, and is generally set to the $\alpha$ th quantile of the distance among the statistics, with $\alpha$ being typically very small (see e.g. Beaumont et al., 2002). With non-informative priors, the original acceptreject algorithm may be very inefficient (Marin et al., 2012). Nevertheless, this issue can be effectively addressed by using more advanced Monte Carlo algorithms, such as MCMC methods (Marjoram et al., 2003), importance sampling (Fearnhead \& Prangle, 2012), sequential or population Monte Carlo approaches (Beaumont et al., 2009; Del Moral et al., 2012; Drovandi \& Pettitt, 2011; Sisson et al., 2007, 2009). Hence, the choice of $\eta(\cdot)$ is a crucial point of $\mathrm{ABC}$. Indeed, what $\mathrm{ABC}$ can achieve at best is $\pi\left(\theta \mid \eta\left(y^{\mathrm{obs}}\right)\right)$, since $\eta(\cdot)$ is rarely sufficient. This loss of information seems to be a necessary price to pay for the access to computable quantities. The idea here is to base the construction of $\eta(\cdot)$ on the score function of a composite likelihood, which is briefly recalled in the next section. 


\subsection{Composite likelihoods}

Let $y=\left(y_{1}, \ldots, y_{n}\right)$ be a realisation of $Y=\left(Y_{1}, \ldots, Y_{n}\right)$, with independent components $Y_{i} \sim f\left(y_{i} ; \theta\right)$, where $y_{i} \in \mathcal{Y} \subseteq \mathbb{R}^{q}$, and let $\left\{A_{1}, \ldots, A_{K}\right\}$ be a set of marginal or conditional events on $\mathcal{Y}$. The composite log-likelihood is defined as (see, e.g., Lindsay, 1988)

$$
c \ell(\theta ; y)=\sum_{i=1}^{n} \sum_{k=1}^{K} w_{k} \log f\left(y \in A_{k} ; \theta\right),
$$

where $w_{k}, k=1, \ldots, K$, are non-negative weights. When the events $A_{k}$ are defined in terms of pairs of bivariate marginal densities $f_{h k}(\cdot, \cdot ; \theta)$, then (2) is called the pairwise log-likelihood and is given by

$$
p \ell(\theta ; y)=\sum_{i=1}^{n} \sum_{\substack{h, k=1 \\ h \neq k}}^{q} w_{h k} \log f_{h k}\left(y_{i h}, y_{i k} ; \theta\right) .
$$

The validity of inference about $\theta$ based on a composite likelihood can be assessed from the standpoint of unbiased estimating functions or the Kullback-Leibler criterion (Cox \& Reid, 2004; Lindsay, 1988; Lindsav et al., 2011; Varin et al., 2011). Under rather broad assumptions (see, for instance, Molenberghs \& Verbeke, 2005), the maximum composite likelihood estimator (MCLE) $\tilde{\theta}$ is the solution of the composite score equation

$$
c \ell_{\theta}(\theta ; y)=\frac{\partial c \ell(\theta ; y)}{\partial \theta}=0 .
$$

The composite score $c \ell_{\theta}(\theta ; y)$ is unbiased, i.e. $E_{\theta}\left\{c \ell_{\theta}(\theta ; Y)\right\}=0$, since it is a linear combination of valid score functions. Moreover, $\tilde{\theta}$ is consistent and approximately normal, with mean $\theta$ and variance

$$
V(\theta)=H(\theta)^{-1} J(\theta) H(\theta)^{-1},
$$

where $H(\theta)=E_{\theta}\left\{-\partial c \ell_{\theta}(\theta ; Y) / \partial \theta^{\top}\right\}$ and $J(\theta)=\operatorname{var}_{\theta}\left\{c \ell_{\theta}(\theta ; Y)\right\}$ are the sensitivity and the variability matrices, respectively. For a full likelihood, $H(\theta)=J(\theta)$ and this is known as the information identity. The matrix $G(\theta)=V(\theta)^{-1}$ is known as the Godambe information, and the sandwich form of $V(\theta)$ is due to the failure of the information identity since, in general, $H(\theta) \neq J(\theta)$. This failure typically implies that the composite likelihood is wrongly too concentrated.

Smith \& Stephenson (2009) discuss the use of the composite likelihood in Bayes' theorem and notice that it may give overly too concentrated posteriors. Pauli et al. (2011) suggest 
to combine a calibrated composite likelihood $c L_{c}(\theta ; y)=\exp \left\{c \ell_{c}(\theta ; y)\right\}$ with a prior $\pi(\theta)$ in order to obtain a calibrated composite posterior

$$
\pi_{c}(\theta \mid y) \propto \pi(\theta) c L_{c}(\theta ; y)
$$

The calibrated composite likelihood is given by

$$
c L_{c}(\theta ; y)=c L(\theta ; y)^{1 / \bar{\omega}}
$$

where $\bar{\omega}=\operatorname{trace}\left\{J(\tilde{\theta}) H(\tilde{\theta})^{-1}\right\} / d$. This calibration approximately adjusts the curvature of the composite likelihood and allows to recover the asymptotic properties of a posterior distribution. Examples of (5) are discussed in Pauli et al. (2011); see also Ribatet et al. (2012) for other types of adjustments.

Bayesian inference based on composite likelihoods leads to composite posteriors, which depend crucially on the calibration adjustment in (6). Since $\bar{\omega}$ is evaluated at $\tilde{\theta}$, this calibration might lead to composite posteriors (5) with overestimated variability (see Section 4).

\section{$3 \quad$ ABC with unbiased estimating functions}

We propose a suitably rescaled composite score function - evaluated at the observed MCLE - as the summary statistic for ABC. This leads to the ABC-cs algorithm, which is introduced and discussed in Section 3.2. The aim of Section 3.1 is to provide a logical motivation for the proposal of Section 3.2, by discussing the ideal, although unrealistic, situation in which a full computable likelihood is available.

\subsection{ABC with score functions}

In this section we show how the score function evaluated at the observed maximum likelihood estimate provides a natural summary statistic for ABC in the, admittedly restrictive, case in which a full likelihood is available. In the following, we first start with a full exponential model and then extend the reasoning to a generic model. 
Consider a full exponential family with density

$$
f(y ; \varphi)=h(y) \exp \left\{\varphi^{\top} s(y)-k(\varphi)\right\}
$$

where $h(y)>0, \varphi$ is the canonical parameter, $s(y)$ is the $d$-dimensional sufficient statistic, and $k(\varphi)$ is the cumulant generating function of $s(y)$. In this case, the obvious summary statistic for $\mathrm{ABC}$ is the minimal sufficient statistic $s(y)$, which gives the exact posterior for $\epsilon \rightarrow 0$ (see, e.g., Blum, 2010). The following proposition shows that the ABC posterior based on a suitably rescaled score function is exact for $\epsilon \rightarrow 0$ and also invariant to reparameterisations.

Proposition 3.1 Let $\ell(\varphi ; y)=\varphi^{\top} s(y)-k(\varphi)$ be the log-likelihood for $\varphi$ based on model (7), and consider as the summary statistic the rescaled score evaluated at a fixed $\varphi_{0}$, that is

$$
\eta\left(y ; \varphi_{0}\right)=B\left(\varphi_{0}\right)^{-1} \ell_{\varphi}\left(\varphi_{0} ; y\right)
$$

where $\ell_{\varphi}(\varphi ; y)=\partial \ell(\varphi ; y) / \partial \varphi=s(y)-\partial k(\varphi) / \partial \varphi$ and $B(\varphi)$ is such that $i(\varphi)=\partial^{2} k(\varphi) /\left(\partial \varphi \partial \varphi^{\top}\right)=$ $B(\varphi) B(\varphi)^{\top}$. Then, the $A B C$ posterior based on $\eta\left(y ; \varphi_{0}\right)$ is exact for $\epsilon \rightarrow 0$ and also invariant to reparameterisations, regardless of the fixed value $\varphi_{0}$.

Proof For any fixed value $\varphi_{0}$, the rescaled score $\eta\left(y ; \varphi_{0}\right)$ is a linear transformation of the minimal sufficient statistic $s(y)$, and thus it is itself minimal sufficient. This proves that the $\mathrm{ABC}$ posterior based on $\eta\left(y ; \varphi_{0}\right)$ is exact for $\epsilon \rightarrow 0$.

Consider the reparametrisation $\theta=\theta(\varphi)$. Let $\bar{\ell}(\theta)=\ell(\varphi(\theta))$ and $\bar{\imath}(\theta)=\varphi_{\theta}^{\top} i(\varphi(\theta)) \varphi_{\theta}$, where $\varphi_{\theta}=\partial \varphi(\theta) / \partial \theta$. The rescaled score is $\bar{\eta}\left(y ; \theta_{0}\right)=\bar{B}\left(\theta_{0}\right)^{-1} \bar{\ell}_{\theta}\left(\theta_{0} ; y\right)$, with $\theta_{0}=\theta\left(\varphi_{0}\right)$, $\bar{\ell}_{\theta}(\theta ; y)=\partial \bar{\ell}(\theta ; y) / \partial \theta$ and $\bar{B}(\theta)$ such that $\bar{B}(\theta) \bar{B}(\theta)^{\top}=\bar{\imath}(\theta)$. Then, since $\bar{B}(\theta)=\varphi_{\theta}^{\top} B(\varphi(\theta))$ and $\bar{\ell}_{\theta}(\theta ; y)=\varphi_{\theta}^{\top} \ell_{\varphi}(\varphi(\theta) ; y)$, it follows that $\bar{\eta}\left(y ; \theta_{0}\right)=\eta\left(y ; \varphi_{0}\right)$. This proves invariance to reparameterisations.

Proposition 3.1 holds for any value of $\varphi_{0}$. In particular, when $\varphi_{0}$ is the observed value of the maximum likelihood estimate (MLE) at the observed data $y^{\text {obs }}$, i.e. $\hat{\varphi}^{\text {obs }}$, we have $\eta\left(y^{\text {obs }} ; \hat{\varphi}^{\text {obs }}\right)=0$. This choice of $\varphi_{0}$ is particularly convenient for a general model $f(y ; \theta)$. Indeed, in this case, at least in principle, we could use an alternative representation of $y$, or equivalently the minimal sufficient statistic based on $y$, given by $(\hat{\theta}, a)$, where $\hat{\theta}$ is the 
MLE and $a$ is an ancillary statistic, which means that its distribution does not depend on $\theta$. Hence, we could replace $f(y ; \theta)$ with $f(\hat{\theta}, a ; \theta)$, and the latter can be factorised as

$$
f(\hat{\theta}, a ; \theta)=f(\hat{\theta} \mid a ; \theta) f(a) .
$$

This means that the likelihood for $\theta$ can be based equivalently on $f(y ; \theta)$ or $f(\hat{\theta} \mid a ; \theta)$. Unfortunately, it may not be easy in general to find $f(\hat{\theta} \mid a ; \theta)$. On the other hand, it is possible to approximate such density through a tangent exponential model at (and near) the fixed value $y^{\text {obs }}$ (Fraser \& Reid, 1995; Reid, 2003, Sect. 3.2). Denoting by $\ell\left(\theta ; y^{\text {obs }}\right)$ the observed loglikelihood, the approximation to the log-likelihood based on the tangent exponential model is

$$
\ell^{\mathrm{TE}}(\theta ; y)=\ell\left(\theta ; y^{\mathrm{obs}}\right)-\ell\left(\hat{\theta}^{\mathrm{obs}} ; y^{\mathrm{obs}}\right)+\left\{\varphi(\theta)-\varphi\left(\hat{\theta}^{\mathrm{obs}}\right)\right\}^{\top} s(y)
$$

where $\hat{\theta}^{\text {obs }}$ is the MLE at the observed data point $y^{\text {obs }}, s(y)=\partial \ell(\theta ; y) /\left.\partial \theta\right|_{\theta=\hat{\theta}^{\text {obs }}}=\ell_{\theta}\left(\hat{\theta}^{\text {obs }} ; y\right)$, and $\varphi(\theta)=\varphi\left(\theta ; y^{\text {obs }}\right)$ is a one-to-one reparameterisation dependent on the observed data $y^{\text {obs }}$ (see also Brazzale et al., 2007, Sect. 8.4.2). The tangent exponential model is a local exponential family model with sufficient statistic $s(y)$ and canonical parameter $\varphi$. It has the same $\log$-likelihood function as the original model at the fixed point $y^{\text {obs }}$, where it also has the same first derivative with respect to $y$.

From Proposition 3.1, the summary statistic for ABC for the tangent exponential model (8) is the rescaled score, where the score is given by

$$
\ell_{\theta}^{\mathrm{TE}}(\theta ; y)=\ell_{\theta}\left(\theta ; y^{\mathrm{obs}}\right)+\varphi_{\theta} s(y)
$$

For $\theta=\hat{\theta}^{\text {obs }}$, (9) reduces to $\varphi_{\theta}\left(\hat{\theta}^{\text {obs }}\right) \ell_{\theta}\left(\hat{\theta}^{\text {obs }} ; y\right)$, i.e. to a linear transformation of the score of the original model. Rescaling (9) then provides invariance to reparameterisation, as in Proposition 3.1. This motivates the use of the score function evaluated at $\hat{\theta}^{\text {obs }}$ as an approximate optimal summary statistic in $\mathrm{ABC}$ for a general model.

Example 1: normal parabola. Let $y=\left(y_{1}, \ldots, y_{n}\right)$ be a random sample from the normal distribution $N\left(\theta, \theta^{2}\right)$, with $\theta>0$. The log-likelihood is

$$
\ell(\theta ; y)=\frac{1}{\theta} \sum_{i=1}^{n} y_{i}-\frac{1}{2 \theta^{2}} \sum_{i=1}^{n} y_{i}^{2}-n \log \theta
$$


where $t(y)=\left(\sum_{i=1}^{n} y_{i}, \sum_{i=1}^{n} y_{i}^{2}\right)$ is the two-dimensional minimal sufficient statistic. The score function is $\ell_{\theta}(\theta ; y)=-\theta^{-2} \sum_{i=1}^{n} y_{i}+\theta^{-3} \sum_{i=1}^{n} y_{i}^{2}-n \theta^{-1}$, which implies that $\hat{\theta}$ is the positive solution of a quadratic equation. The expected information is $i(\theta)=3 n / \theta^{2}$, and the rescaled score is $\eta\left(y ; \hat{\theta}^{\text {obs }}\right)=\hat{\theta}^{\text {obs }} \ell_{\theta}\left(\hat{\theta}^{\text {obs }} ; y\right) / \sqrt{3 n}$.

As an illustration we use a sample of size $n=50$ generated from the model, with $\theta=5$ and with a uniform prior in $(0,15)$. We consider three instances of the ABC Algorithm 1, with distance $\rho(v, w)=\|v-w\|_{1}$ and with summary statistics given, respectively, by $t(y)$, $\eta\left(y ; \hat{\theta}^{\text {obs }}\right)$, and also a one-to-one transformation of the minimal sufficient statistic $t(y)$, that is $t_{1}(y)=\left(\bar{y}, \sqrt{s^{2}}\right)$, i.e. the sample mean and the standard deviation. In all three cases, we use the same sample of $10^{7}$ values generated from the prior and in each case we choose the threshold $\epsilon$ as the quantile of level $0.1 \%$ of the observed distances, thus accepting $10^{4}$ values. These $\epsilon$ values are, respectively, 31.264, 0.02 and 0.237 . These values are not directly comparable, since the three statistics are not on the same scale. A possibility would be to suitably standardize $t(y)$ and $t_{1}(y)$, but such a standardisation is not obvious in general. On the other hand, the statistic $\eta\left(y ; \hat{\theta}^{\text {obs }}\right)$ is rescaled using the variability of the score. For vector parameters this rescaling also takes into account the correlation among the components of the statistic.
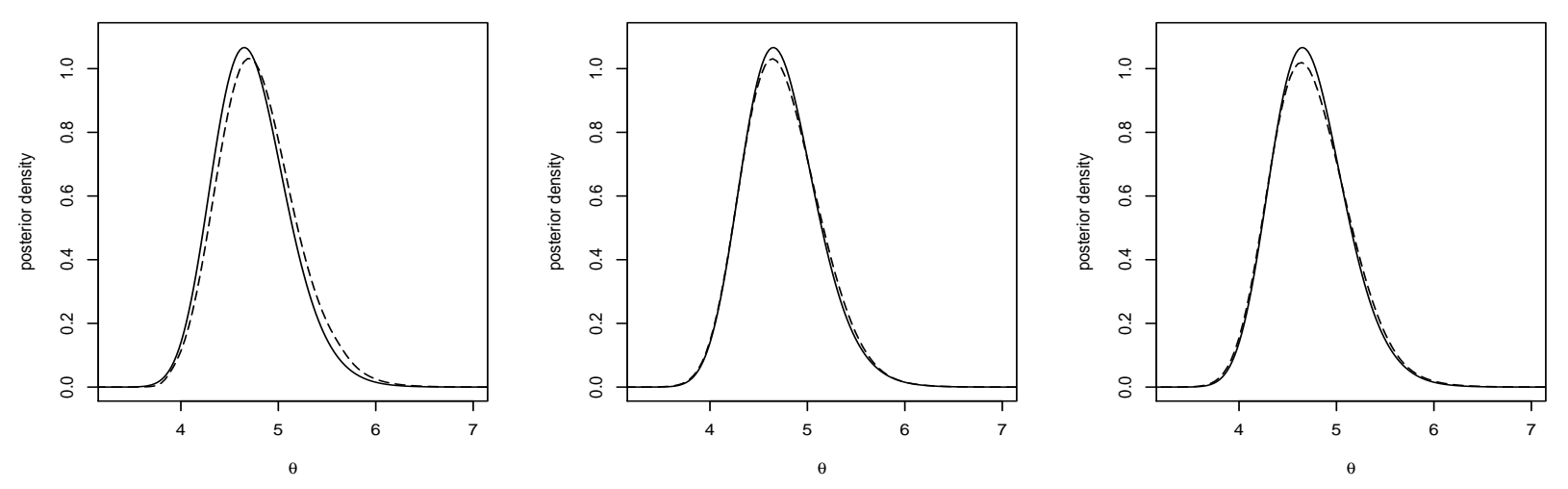

Figure 1: Normal parabola. In all panels the solid line corresponds to the exact posterior, while the dashed lines correspond to ABC approximations using $t(y)$ (left panel), $t_{1}(y)$ (central panel), and $\eta\left(y ; \hat{\theta}^{\text {obs }}\right)$ (right panel).

Figure 1 shows the three approximations compared with the exact posterior. The two 
versions of the $\mathrm{ABC}$ with the minimal sufficient statistic gave quite different results, with the one with $t(y)$ leading to the worst accuracy. This is likely due to the fact that the the two components of $t(y)$ are on different scales. On the other hand, the ABC with the one-dimensional summary statistic $\eta\left(y ; \hat{\theta}^{\text {obs }}\right)$, which is not sufficient for this model, gave an approximation to the posterior with accuracy comparable with ABC with the minimal sufficient statistic $t_{1}(y)$.

In order to check that this behaviour is not due to the particular simulated dataset, we consider the same experiment on 50 different datasets, and for each posterior we compute the Kullback-Leibler (KL) divergence among the exact and the three approximate posteriors. A plot of the log-KL divergences is given in Figure 2, which confirms the good agreement of $\mathrm{ABC}$ with $\eta\left(y ; \hat{\theta}^{\mathrm{obs}}\right)$ and $\mathrm{ABC}$ with the minimal sufficient statistic $t_{1}(y)$, but not with the minimal sufficient statistic $t(y)$.

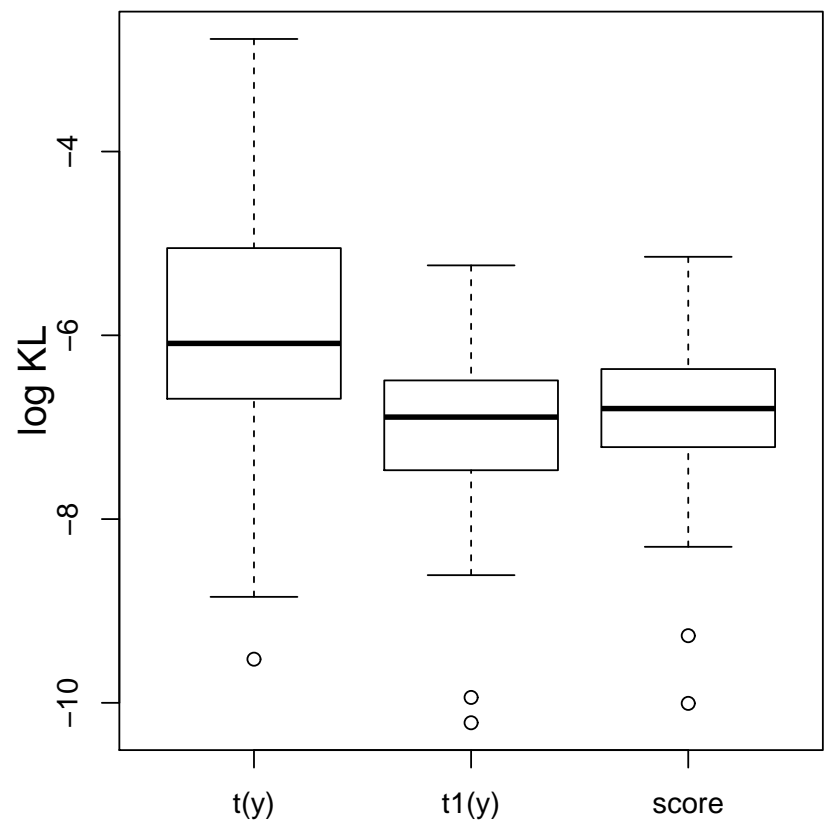

Figure 2: Kullback-Leibler divergences (logarithmic scale) among the exact and ABC posteriors using $t(y), t_{1}(y)$ and $\eta\left(y ; \hat{\theta}^{\text {obs }}\right)$, over 50 replicated datasets for the normal parabola.

Remark 1. From the point of view of the likelihood principle, the different performances of 
the $\mathrm{ABC}$ algorithm with both versions of the minimal sufficient statistic in Example 1 is unpleasant. Indeed, $t(y)$ and $t_{1}(y)$ lead to the same likelihood function and posterior distribution, but the corresponding ABC approximations could be remarkably different. Hence, transforming the summary statistic may have a great impact on the quality of the ABC approximation. Finding the right transformation may not be straightforward, especially when the summary statistic is high-dimensional. This issue has already been recognised in the ABC literature. For instance, Jung \& Marjoram (2011) propose to weight the components of the summary statistic using a genetic algorithm, though the method seems computationally quite intensive. On the contrary, since the likelihood and the score functions are not affected by one-to-one transformations of the data, or of the minimal sufficient statistic, ABC with $\eta\left(y ; \hat{\theta}^{\text {obs }}\right)$ is invariant with respect to such transformations. This invariance to data transformations adds to the parameterisation invariance proved in Proposition 3.1 ,

Remark 2. Although the choice of the distance function $\rho(\cdot, \cdot)$ in the $\mathrm{ABC}$ algorithm is arbitrary, when considering the Euclidean distance we have

$$
\rho\left(\eta\left(y ; \hat{\theta}^{\mathrm{obs}}\right), \eta\left(y^{\mathrm{obs}} ; \hat{\theta}^{\mathrm{obs}}\right)\right)=\left\|\eta\left(y ; \hat{\theta}^{\mathrm{obs}}\right)\right\|_{2}^{1 / 2}=\left\{\ell_{\theta}\left(\hat{\theta}^{\mathrm{obs}} ; y\right)^{\top} i\left(\hat{\theta}^{\mathrm{obs}}\right)^{-1} \ell_{\theta}\left(\hat{\theta}^{\mathrm{obs}} ; y\right)\right\}^{1 / 2}
$$

which is the square root of the score test statistic computed in $\hat{\theta}^{\text {obs }}$, based on data $y$.

Despite the good properties of $\mathrm{ABC}$ with the score function, in typical applications of the $\mathrm{ABC}$ method the likelihood function is intractable, and therefore the same is true for the score function. This motivates the extension to composite likelihoods proposed in the next section.

\subsection{ABC with composite score function}

When dealing with complex models, possible surrogates of the unavailable full likelihood are given by composite likelihoods. Extending the results of the previous section, we propose the rescaled composite score function as a summary statistic in ABC. This defines an algorithm, called ABC-cs. In terms of the ABC Algorithm 1, ABC-cs replaces the matching condition

$$
\rho\left(\eta(y), \eta\left(y^{\mathrm{obs}}\right)\right) \leq \epsilon,
$$


with

$$
\rho\left(\eta_{c}\left(\tilde{\theta}^{\mathrm{obs}} ; y\right), \eta_{c}\left(\tilde{\theta}^{\mathrm{obs}} ; y^{\mathrm{obs}}\right)\right) \leq \epsilon
$$

where $\tilde{\theta}^{\text {obs }}$ is the MCLE computed with $y^{\text {obs }}$ and

$$
\eta_{c}\left(\tilde{\theta}^{\mathrm{obs}} ; y\right)=B_{c}\left(\tilde{\theta}^{\mathrm{obs}}\right)^{-1} c \ell_{\theta}\left(\tilde{\theta}^{\mathrm{obs}} ; y\right)
$$

is the rescaled composite score, with $B_{c}(\theta)$ such that $J(\theta)=B_{c}(\theta) B_{c}(\theta)^{\top}$. Since $c \ell_{\theta}\left(\tilde{\theta}^{\text {obs }} ; y^{\text {obs }}\right)=$ 0 , in (10) we only need to evaluate $\eta_{c}\left(\tilde{\theta}^{\mathrm{obs}} ; y\right)$.

The following theorem shows that the proposed ABC-cs algorithm gives an approximate posterior distribution with the correct curvature, in the sense discussed at the end of Section 2.2, even if the rescaled composite score function (11), unlike the full score function, does not satisfy the information identity.

Theorem 3.2 The ABC-cs algorithm with the rescaled composite score statistic $\eta_{c}\left(\tilde{\theta}^{\text {obs }} ; y\right)$, as $\varepsilon \rightarrow 0$, leads to an approximate posterior distribution with the correct curvature and is also invariant to reparameterisations.

Proof In order to recover the information identity, and thus the correct curvature, it is necessary to consider the adjusted composite score function (see, e.g., Pace \& Salvan, 1997, Chap. 4)

$$
g(\theta ; y)=H(\theta) J(\theta)^{-1} c \ell_{\theta}(\theta ; y)=A(\theta) c \ell_{\theta}(\theta ; y)
$$

Indeed, for $g(\theta ; y)$ we have

$$
J_{g}(\theta)=\operatorname{var}_{\theta}\{g(\theta ; Y)\}=A(\theta) \operatorname{var}_{\theta}\left\{c \ell_{\theta}(\theta ; Y)\right\} A(\theta)^{\top}=G(\theta)
$$

and, using $E_{\theta}\left\{c \ell_{\theta}(\theta ; Y)\right\}=0$,

$$
H_{g}(\theta)=E_{\theta}\left\{-\frac{\partial}{\partial \theta^{\top}} g(\theta ; Y)\right\}=-A(\theta) E_{\theta}\left\{\frac{\partial}{\partial \theta^{\top}} c \ell_{\theta}(\theta ; Y)\right\}=G(\theta) .
$$

Since $H_{g}(\theta)=J_{g}(\theta)=G(\theta)$, the adjusted composite score $g(\theta ; y)$ satisfies the information identity as a proper score function and, since $|A(\theta)| \neq 0, g(\theta ; y)=0$ leads to the same solution $\tilde{\theta}$ of the estimating equation $c \ell_{\theta}(\theta ; y)=0$. 
The ABC-cs algorithm should then be based on the rescaled version of $g(\theta ; y)$, given by

$$
\eta_{g}\left(\tilde{\theta}^{\mathrm{obs}} ; y\right)=B_{g}\left(\tilde{\theta}^{\mathrm{obs}}\right)^{-1} g\left(\tilde{\theta}^{\mathrm{obs}} ; y\right)
$$

where $B_{g}(\theta)=H(\theta)\left\{B_{c}(\theta)^{\top}\right\}^{-1}$. Indeed,

$$
G(\theta)=H(\theta) J(\theta)^{-1} H(\theta)=H(\theta)\left\{B_{c}(\theta)^{\top}\right\}^{-1} B_{c}(\theta)^{-1} H(\theta)
$$

However, it is straightforward to see that

$$
\eta_{g}(\theta ; y)=B_{g}(\theta)^{-1} g(\theta ; y)=B_{c}(\theta)^{\top} H(\theta)^{-1} H(\theta) J(\theta)^{-1} c \ell_{\theta}(\theta ; y)=\eta_{c}(\theta ; y)
$$

This proves that the use of $\eta_{c}\left(\tilde{\theta}^{\text {obs }} ; y\right)$ as a summary statistic for ABC leads to an approximate posterior with the correct curvature.

The proof of invariance to reparameterisation follows the same steps as in Proposition 3.1 .

An advantage of $\mathrm{ABC}-\mathrm{cs}$ is that the rescaled composite score statistic has the same dimension as $\theta$. Moreover, since the score statistic is obtained from the composite loglikelihood by just taking the first derivative, it is easily computed, especially when it is analytically available. An apparent drawback of (11) is the implicit dependence of the ABCcs algorithm on $J(\theta)$. However, only $J\left(\tilde{\theta}^{\text {obs }}\right)$ is needed, and this quantity can be easily approximated with a preliminary Monte Carlo simulation from the model with $\theta=\tilde{\theta}^{\text {obs }}$, with few hundred replications (Cattelan \& Sartori, 2014). Finally, note that even in this case, the squared Euclidean distance gives the composite score test statistic evaluated in $\tilde{\theta}^{\text {obs }}$, based on data $y$.

The ABC-cs algorithm delivers an approximate posterior distribution which does not need calibration, whereas Bayesian composite posteriors depend crucially on such quantities. Moreover, even when rescaled, the Bayesian composite posterior (5) often leads to less accurate results, as also shown in the examples of Section 4 and in the application of Section 5.

As a final remark, we note that the proposal of this paper is not providing an automatic summary statistic for $\mathrm{ABC}$, in the sense that an appropriate choice of composite likelihood 
for the problem under investigation must be made. The composite likelihood may be difficult, if not impossible, to define in some applications (see, for instance, the non-Markovian queueing model analysed by Heggland \& Frigessi, 2004), while in other situations there could be different competing composite likelihoods available for the same model. The latter case will be addressed more in detail in the final discussion. The point here is that, when there is at least one composite likelihood available, it is usually defined starting from relevant stochastic features of the model and therefore the summary statistic based on the composite score will automatically incorporate these features. Moreover, there is an extensive, and growing, frequentist literature on composite likelihoods (see, for instance, the review by Varin et al., 2011), that can be used to guide the choice of a sensible composite likelihood in specific classes of models.

\section{Examples}

In the examples below we use composite marginal likelihood functions (Cox \& Reid, 2004), although different model structures might lead to different choices of suitable composite like-

lihoods. We use the Godambe information $G\left(\tilde{\theta}^{\text {obs }}\right)$ as a precision matrix for both $\mathrm{ABC}$ and ABC-cs with importance sampling. Note that ABC with MCMC or Sequential Monte Carlo (SMC) methods requires a similar precision matrix, which in practice is estimated by considering preliminary runs of $\mathrm{ABC}$ (in the case of MCMC) or from a previous population of ABC particles (in the case of SMC). The $\mathrm{R}$ code for the examples of this section and for the application in Section 5 can be found in the Supplementary Material.

\section{Example 2: equi-correlated normal model}

This example focuses on inference based on the pairwise log-likelihood (3) for the parameters of an equi-correlated multivariate normal distribution, with mean vector $\mu$ and covariance matrix $\Sigma_{r s}=\rho \sigma^{2}$, for $r \neq s$, and $\Sigma_{r r}=\sigma^{2}, r, s=1, \ldots, q$. For this model, $\tilde{\theta}$ is fully efficient, the sufficient statistic is three-dimensional and is the same for both the full and pairwise 
likelihoods (Pace et al., 2011). The pairwise log-likelihood (13) for $\theta=\left(\mu, \sigma^{2}, \rho\right)$ is

$$
\begin{aligned}
p \ell(\theta ; y)= & -\frac{n q(q-1)}{2} \log \sigma^{2}-\frac{n q(q-1)}{4} \log \left(1-\rho^{2}\right)-\frac{q-1+\rho}{2 \sigma^{2}\left(1-\rho^{2}\right)} S S_{W} \\
& -\frac{q(q-1) S S_{B}+n q(q-1)(\bar{y}-\mu)^{2}}{2 \sigma^{2}(1+\rho)},
\end{aligned}
$$

where $S S_{W}=\sum_{i=1}^{n} \sum_{r=1}^{q}\left(y_{i r}-\bar{y}_{i}\right)^{2}, S S_{B}=\sum_{i=1}^{n}\left(\bar{y}_{i}-\bar{y}\right)^{2}, \bar{y}_{i}=\sum_{r=1}^{q} y_{i r} / q$ and $\bar{y}=$ $\sum_{i=1}^{n} \sum_{r=1}^{q} y_{i r} /(n q)$. For the expression of the score function see Pace et al. (2011, p. 145).

We assume that the components of the parameter $\omega=(\mu, \tau, \kappa)$, with $\tau=\log \sigma^{2}$ and $\kappa=\operatorname{logit}(\{\rho(q-1)+1\} / q)$, are independent, with $N(0,100)$ marginal prior distributions.

As an illustration, we use a sample of $n=30$ drawn from the model with $q=50$, $\mu=0, \sigma^{2}=1$ and $\rho=0.5$. For $\mathrm{ABC}$ we used the sufficient statistic $\left(\bar{y}, \sqrt{S S_{B}}, \sqrt{S S_{w}}\right)$, which gave better results than the alternative form $\left(\bar{y}, S S_{B}, S S_{w}\right)$, while for ABC-cs the summary statistic is given by (11). The simulation from the ABC and ABC-cs posteriors is performed with importance sampling, where the importance function is the multivariate $t$-student distribution with 5 degrees of freedom, centred at $\tilde{\theta}^{\text {obs }}$ and with scale matrix equal to $5 V\left(\tilde{\theta}^{\text {obs }}\right)$. We consider $10^{3}$ final samples obtained with $\epsilon$ fixed at the $0.1 \%$ quantile of the observed distances. Finally, in order to get rid of the importance weights, here and elsewhere, we consider resampling with replacement of the simulated values.

Results are compared also with the pairwise posterior

$$
\pi_{p l}(\theta \mid y) \propto \pi(\theta) \exp \{p \ell(\theta ; y)\}
$$

with the pairwise posterior (5) based on the calibrated pairwise likelihood and with the posterior distribution based on the full likelihood, approximated by a random walk Metropolis.

The boxplots of the marginal posterior approximations are shown in Figure 3, which highlights several interesting features. The posterior (12) appears wrongly too concentrated (see also Pauli et al., 2011; Ribatet et al., 2012; Smith \& Stephenson, 2009), whereas the calibrated pairwise posterior (5) may have the opposite problem. Indeed, while the marginal calibrated pairwise posteriors of $\mu$ and $\tau$ are quite similar to the full posterior (MCMC), the marginal calibrated pairwise posterior of $\kappa$ shows higher dispersion than the corresponding marginal based on the full likelihood. On the other hand, ABC-cs and ABC marginal 

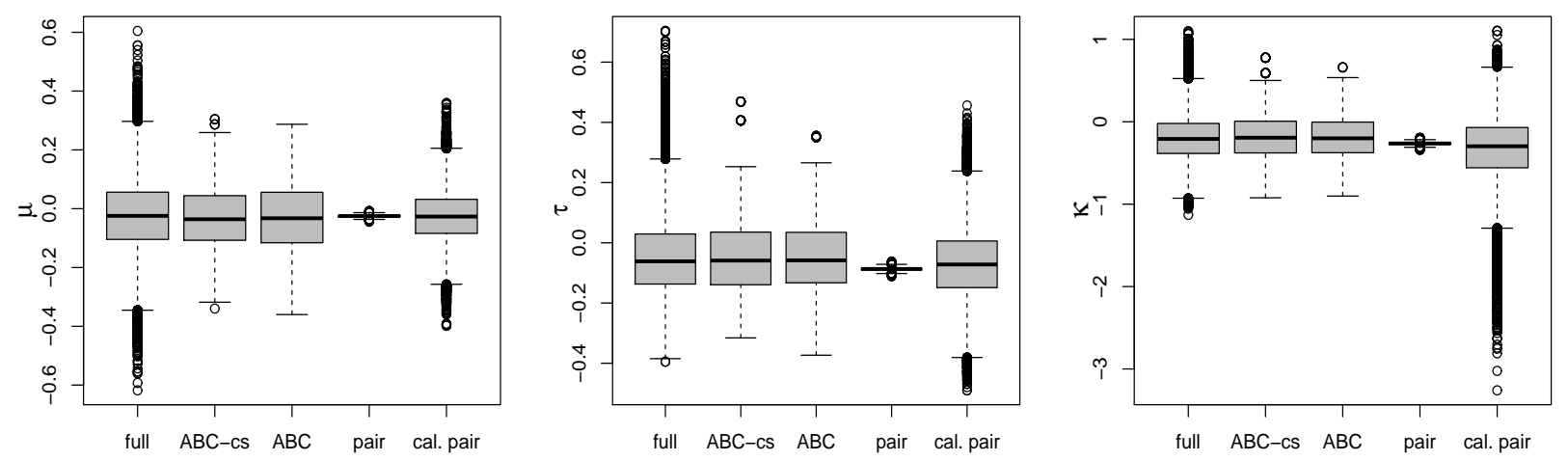

Figure 3: Equi-correlated normal model. ABC-cs posterior compared with the full, the pairwise (pair), the calibrated pairwise (cal. pair) and the $\mathrm{ABC}$ posteriors.

posteriors are all quite similar to the full posterior. This is not surprising, since the model is a full exponential family of order three and $\mathrm{ABC}$ uses exactly the sufficient statistic as summary statistic. Moreover, even the pairwise likelihood has exponential form, with the same sufficient statistic. This implies that the pairwise score function is proportional to the score function of the full model (Kenne Pagui et al., 2015) and the latter would lead again to the sufficient statistic (see Section 3.1).

We also compare the posterior means of the full, ABC, ABC-cs and the calibrated pairwise posteriors in a simulation study, over 100 Monte Carlo trials. The data are generated from the model with $\mu=0, \sigma^{2}=1, \rho=0.2$. Figure 4 indicates that ABC and ABC-cs posterior means are quite similar to the full posterior mean, as expected from Proposition 3.1. On the contrary, for the transformed correlation parameter $\kappa$ the mean of the calibrated pairwise posterior can perform poorly. The behavior of the calibrated pairwise likelihood is due to the fact that the overall rescaling, computed at the mode, does not generally guarantee accuracy in the tails. Simulations for other parameter configurations can be found in the Supplementary Material.

\section{Example 3: multivariate probit model}

The pairwise likelihood is particularly useful for modelling correlated binary outcomes, as discussed in Le Cessie \& van Houwelingen (1994). Correlated binary data typically arise in the context of repeated measurements on the same individual. Standard likelihood analysis 

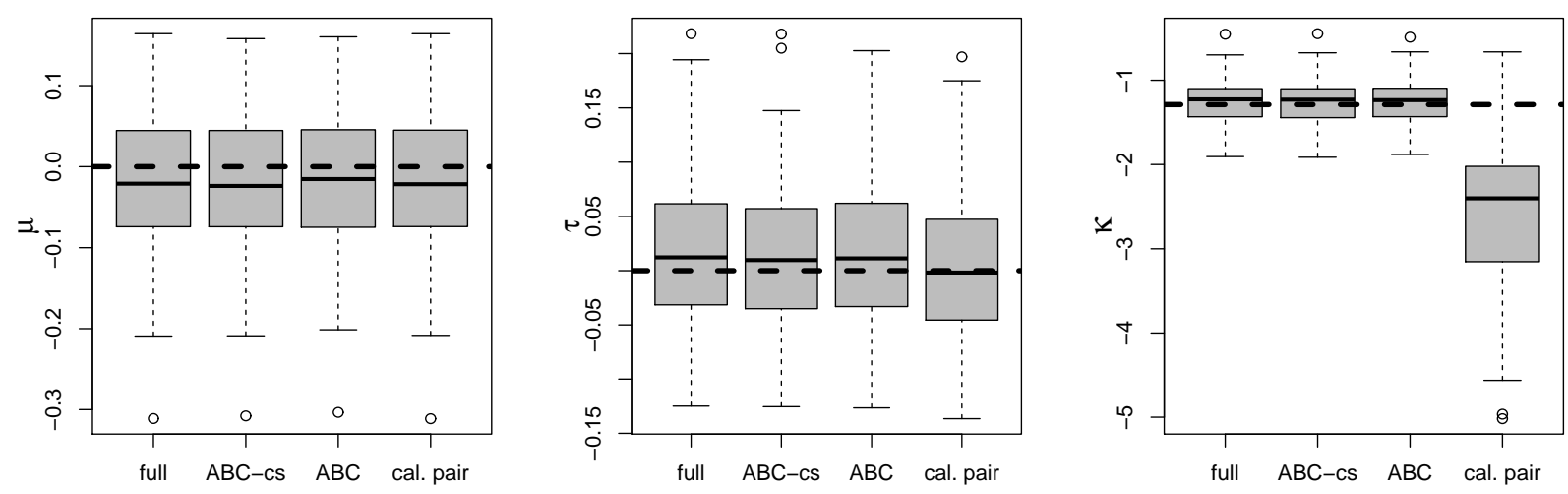

Figure 4: Equi-correlated normal model. Simulation study based on 100 Monte Carlo trials, with $\mu=0, \sigma=1(\tau=0)$ and $\rho=0.2(\kappa \approx-1.15)$. The dashed horizontal lines represent the true parameter values.

in these contexts may be difficult because it involves multivariate integrals whose dimension equals the cluster sizes.

Let us focus on a multivariate probit model with constant cluster sizes. In particular, let $S_{i}=\left(S_{i 1}, \ldots, S_{i q}\right)$ be a latent normal random variable with mean $\gamma_{i}$ and covariance matrix $\Sigma$, with $\Sigma_{h h}=1+\sigma^{2}, \Sigma_{h k}=\sigma^{2}, h \neq k, h, k=1, \ldots, q$. We assume $\gamma_{i}=X_{i} \beta$, where $\beta$ is a vector of unknown regression coefficients and $X_{i}$ is the design matrix for unit $i, i=1, \ldots, n$, Then, the observed data $Y_{i h}$ is equal to 1 if $S_{i h}>0$, and 0 otherwise.

The full likelihood is computationally cumbersome since it entails calculation of multiple integrals of a $q$-variate multivariate normal distribution. On the other hand, the pairwise log-likelihood is

$$
p \ell\left(\beta, \sigma^{2} ; y\right)=\sum_{i=1}^{n} \sum_{h=1}^{q-1} \sum_{k=h+1}^{q} \log \operatorname{Pr}\left(Y_{i h}=y_{i h}, Y_{i k}=y_{i k} ; \beta, \rho\right), \quad y_{i h}, y_{i k} \in\{0,1\},
$$

where, for instance, $\operatorname{Pr}\left(Y_{i h}=1, Y_{i k}=1 ; \beta, \rho\right)=\Phi_{2}\left(\gamma_{i h}, \gamma_{i k} ; \rho\right)$ is the standard bivariate normal distribution, with correlation $\rho=\sigma^{2} /\left(1+\sigma^{2}\right)$ and with $\gamma_{i h}=x_{i h} \beta / \sqrt{1+\sigma^{2}}$ the $h$ th component of $\gamma_{i}$ (see, for instance, Cattelan \& Sartori, 2014).

As an example, we consider data generated with $\beta_{0}=0.5, \beta_{1}=1.5, \sigma^{2}=1, n=30$ and $q=10$, where $\beta_{0}$ is the intercept and $\beta_{1}$ the coefficient of a covariate generated from the uniform distribution in $(-1,1)$. For the parameter $\theta=\left(\beta_{0}, \beta_{1}, \log \sigma^{2}\right)$ a trivariate normal 
prior with independent components $N(0,100)$ is assumed. For $\mathrm{ABC}$ we take the counts at each time point $h, h=1, \ldots, q$, as a $q$-dimensional summary statistic. Hence, the absolute norm of the difference among the statistics is $\sum_{h=1}^{q}\left|\sum_{i=1}^{n}\left(y_{i h}^{\text {obs }}-y_{i h}\right)\right|$. Other choices of the summary statistic led to less accurate results. For ABC-cs, we consider the rescaled pairwise score, evaluated at $\tilde{\theta}^{\text {obs }}$. The matrices $J\left(\tilde{\theta}^{\text {obs }}\right)$ and $H\left(\tilde{\theta}^{\text {obs }}\right)$ were computed by simulation with 1000 datasets taken from the model with $\theta=\tilde{\theta}^{\text {obs }}$. We consider $10^{3}$ final samples drawn from the $\mathrm{ABC}$ and $\mathrm{ABC}$-cs posteriors after fixing $\epsilon$ to the $0.1 \%$ quantile of the observed distances. The sampling is done via importance sampling, with a multivariate $t$-student importance density, with 5 degrees of freedom, centred at $\tilde{\theta}^{\text {obs }}$ and with scale matrix equal to $5 V\left(\tilde{\theta}^{\text {obs }}\right)$. We compare the results also with the full posterior approximated by the MCMC method of Chib \& Greenberg (1998), and with the pairwise and the calibrated pairwise posteriors approximated by usual MCMC. All MCMC approximations are based on $3 \times 10^{4}$ posterior samples, of which the first 5000 values are discarded.

Figure 5 shows that the $\mathrm{ABC}-\mathrm{cs}$ method gives a better approximation than $\mathrm{ABC}$ with the chosen summary statistic, when compared to the full posterior computed by MCMC. On the other hand, the non calibrated pairwise posterior is overly concentrated, whereas the calibrated pairwise posteriors of $\beta_{0}$ and $\log \sigma^{2}$ seem too dispersed.
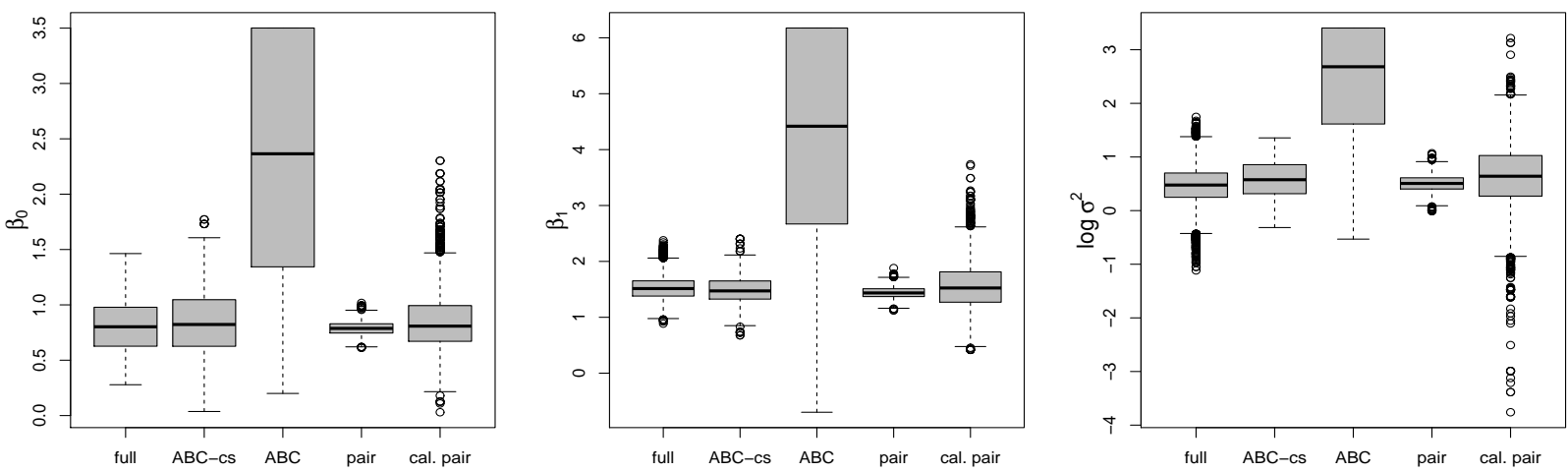

Figure 5: Multivariate probit model. ABC-cs posterior compared with the ABC, the pairwise (pair), the calibrated pairwise (cal. pair) and the full posteriors.

A simulation study is conducted over 100 Monte Carlo samples, where the data are simulated as above, with $\beta_{0}=0.5, \beta_{1}=1.5, n=30, q=10$ and $\sigma^{2}=4$. For each simulated dataset, we consider the mean of the $\mathrm{ABC}, \mathrm{ABC}-\mathrm{cs}$, calibrated pairwise and full posteriors. 
Figure 6 highlights that the mean of the $\mathrm{ABC}$ posterior shows more variability and more bias with respect to the true value (dashed line). On the other hand, the ABC-cs mean is more accurate than the mean of the calibrated pairwise posterior and is in reasonable agreement with the mean of the full posterior. See the Supplementary Material for additional simulation results with different parameter configurations.
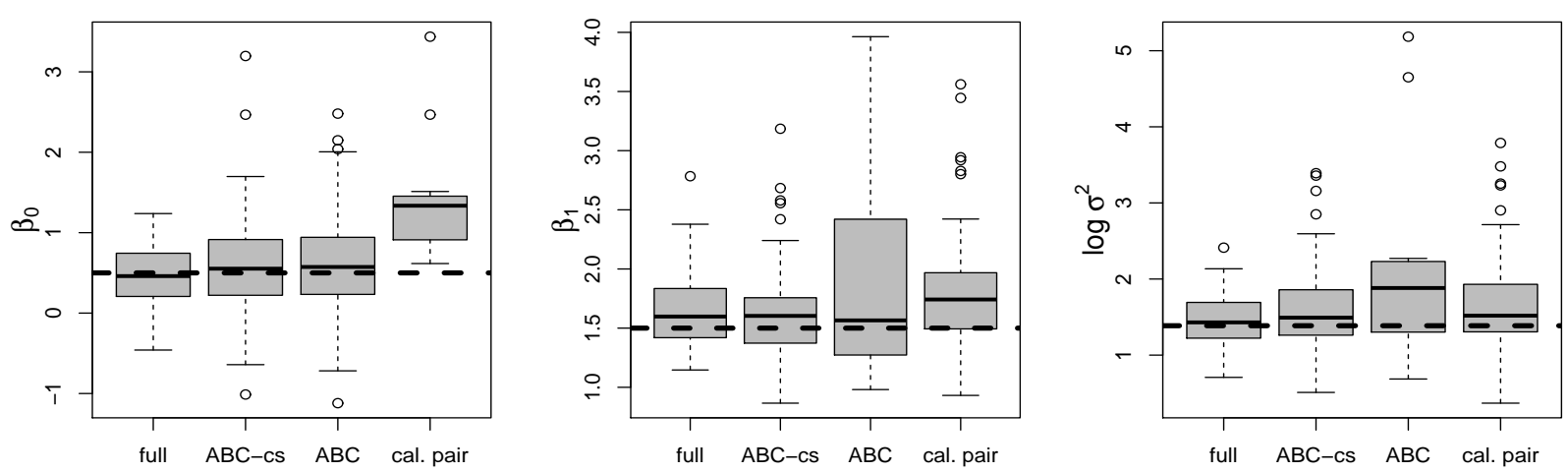

Figure 6: Multivariate probit model. Simulations based on 100 Monte Carlo trials, with $\beta_{0}=0.5$, $\beta_{1}=1$ and $\sigma^{2}=4\left(\log \sigma^{2}=1.39\right)$.

\section{$5 \quad$ Spatial extremes}

Understanding and modelling the behaviour of natural phenomena such as heat waves, heavy rainfall or air pollution can be of interest for climate, social and statistical scientists, to stakeholders such as insurance companies and public health officials. It is therefore important to have useful statistical methods for modelling these extreme occurrences and assessing their possible consequences and impacts.

As these phenomena materialize in spatio-temporal contexts, a natural approach to their modelling is through the theory of max-stable processes (see De Haan, 1984; Kabluchko et al., 2009; Schlather, 2002), an infinite-dimensional extension to multivariate extreme value theory. Max-stable modelling has the potential advantage of accounting for spatial dependence of extremes in a way that is consistent with the classical extreme-value theory, but is much less well developed than other competitive approaches (Davison et al., 2012). Some appli- 
cations to rainfall data can be found in Buishand et al. (2008), Smith \& Stephenson (2009), Padoan et al. (2010), Davison et al. (2012), Ribatet et al. (2012), to temperature data in Davison \& Gholamrezaee (2012), and to snowfall data in Blanchet \& Davison (2011).

Here we focus on the popular max-stable process introduced by Smith (1990). Full description of this model can be found in Padoan et al. (2010) and Davison et al. (2012), to which we refer for the details. The bivariate marginal distribution of Smith's model at spatial coordinates $t_{k}, t_{l} \in \mathbb{R}^{2}$, for $k \neq l=1, \ldots, q$, with $q$ being the number of spatial locations, is $\operatorname{Pr}\left\{Z\left(t_{k}\right) \leq z_{k}, Z\left(t_{l}\right) \leq z_{l}\right\}=\exp \left[-\frac{1}{z_{k}} \Phi\left(\frac{a(h)}{2}+\frac{1}{a(h)} \log \frac{z_{l}}{z_{k}}\right)-\frac{1}{z_{l}} \Phi\left(\frac{a(h)}{2}+\frac{1}{a(h)} \log \frac{z_{k}}{z_{l}}\right)\right]$ where $h=\left(t_{l}-t_{k}\right), a(h)=\left(h^{\top} \Sigma^{-1} h\right)^{1 / 2}, \Sigma$ is the covariance matrix of the process with variances $\sigma_{1}^{2}, \sigma_{2}^{2}>0$ and covariance $\sigma_{12}$. The corresponding density function is obtained by straightforward differentiation (see, e.g., Padoan et al., 2010).

An expression for the trivariate marginal density of Smith's model is derived by Genton et al. (2011). However, there is no closed form expression for marginal densities of dimension greater than three and so the full likelihood is intractable. Pairwise likelihood inference is therefore a natural approach in this context, and it was first advocated by Padoan et al. (2010); see also Blanchet \& Davison (2011), Sang \& Genton (2014), Ribatet et al. (2012) and Smith \& Stephenson (2009). Although the triplewise likelihood can be more efficient than the pairwise likelihood (Genton et al., 2011), for processes typically used in applications the efficiency gains are not striking and therefore the pairwise likelihood provides a good compromise between statistical and computational efficiency (Huser \& Davison, 2013).

The extremal dependence of Smith's model, and in general for other types of max-stable processes, is typically studied through the so called extremal coefficient (Smith, 1990). In practice, due to high-dimensional distributional complexity the extremal coefficient is limited to pairwise components. Specifically, for Smith's model such extremal coefficient is $\delta(h)=$ $2 \Phi(a(h) / 2)$, and the range of the spatial dependence is thus completely governed by $\Sigma$.

Given the data $y_{1}, \ldots, y_{n}$, assumed to be $n$ independent replications of the random vector $Y_{i} \in \mathbb{R}^{q}, i=1, \ldots, n$, with marginal unit Fréchet distribution, Padoan et al. (2010) fit Smith's model by maximising the associated pairwise likelihood. For the generic pair of sites 
$k, l(k \neq l), k, l=1, \ldots, q$, and observation $i$, the pairwise log-likelihood is

$$
p \ell\left(\theta ; y_{i k}, y_{i l}\right)=A+\log (B C+D)+\log E,
$$

with

$$
\begin{gathered}
A=-\frac{\Phi(w(h))}{z_{i k}}-\frac{\Phi(v(h))}{z_{i l}}, \quad B=\frac{\Phi(w(h))}{z_{i k}^{2}}+\frac{\phi(w(h))}{a(h) z_{i k}^{2}}-\frac{\phi(v(h))}{a(h) z_{i k} z_{i l}}, \\
C=\frac{\Phi(v(h))}{z_{i l}^{2}}+\frac{\phi(v(h))}{a(h) z_{i l}^{2}}-\frac{\phi(w(h))}{a(h) z_{i k} z_{i l}}, \quad D=\frac{v(h) \phi(w(h))}{a(h)^{2} z_{i k}^{2}}+\frac{w(h) \phi(v(h))}{a(h)^{2} z_{i k} z_{i l}^{2}}, \\
E=\frac{1}{\lambda_{k} \lambda_{t}}\left(1+\xi_{k} \frac{y_{i k}-\mu_{k}}{\lambda_{k}}\right)_{+}^{1 / \xi_{k}-1}\left(1+\xi_{l} \frac{y_{i l}-\mu_{l}}{\lambda_{l}}\right)_{+}^{1 / \xi_{l}-1},
\end{gathered}
$$

$w(h)=a(h) / 2+\log \left(z_{i l} / z_{i k}\right) / a(h), v(h)=a(h)-w(h)$ and $a_{+}=\max \{0, a\}$. Notice that $E$ is essentially the Jacobian due to the standardisation from the observed data $y_{i k}$ to unit Fréchet $z_{i k}$, and $\mu_{k}, \lambda_{k}>0$ and $\xi_{k}$ are continuous functions, that represent respectively location, scale and shape at site $k$.

Following Padoan et al. (2010), $\mu_{k}$ and $\lambda_{k}$ are assumed as response surfaces, that is $\mu_{k}=$ $X_{k}^{\mu} \beta^{\mu}, \lambda_{k}=X_{k}^{\lambda} \beta^{\lambda}$, where $X_{k}^{\mu}$ and $X_{k}^{\lambda}$ are vectors of covariates for location $k$, whereas $\beta^{\mu} \in \mathbb{R}^{p^{\mu}}$ and $\beta^{\lambda} \in \mathbb{R}^{p^{\lambda}}$ are unknown regression parameters $(k=1, \ldots, q)$. Moreover, for simplicity we assume equal shape among the $q$ locations, e.g. $\xi_{k}=\xi$, for all $k$. The parameter of this model is $\theta=\left(\sigma_{1}^{2}, \sigma_{12}, \sigma_{2}^{2}, \beta^{\mu}, \beta^{\lambda}, \xi\right)$. Other possible models can be constructed by considering spline functions instead of linear regression functions but, for simplicity, here we focus on the latter.

The fitting of max-stable processes for extremes with ABC methods has been first proposed by Erhardt \& Smith (2012). In particular, they transform the data to unit Gumbel, where the marginal parameters are estimated separately by fitting the Generalised Extreme Value (GEV) distribution at each location by maximum likelihood estimation, and successively estimate the dependence parameters using ABC. The summary statistic proposed by Erhardt \& Smith (2012) is the least square fit of the residuals among the empirical and theoretical pairwise or triplewise madogram. However, ABC-cs allows to estimate jointly both the marginal GEV and tail dependence parameters. Moreover, the rescaled composite score is not computationally as demanding as the summary statistic of Erhardt \& Smith (2012), which at each simulated data requires a least square fit and a scalar numerical integration. 
We illustrate ABC-cs using summer (June to August) maximum daily rainfall data at $q=79$ rain gauging stations located in the north of the Alps and east of the Jura mountains in Switzerland. The dataset is provided by the national meteorological service (MétéoSuisse) and comprises $n=49$ yearly observations which were derived from daily precipitation data from 1962 to 2008 (a reduced version of this dataset is used also by Davison et al., 2012 and Sang \& Genton, 2014). The full dataset can be found in the R package SpatialExtremes (Ribatet et al., 2013). An exploratory data analysis reveals that there is some variation in the precipitation with latitude and longitude, which suggests that the process may be anisotropic; see also Davison et al. (2012) for a more in-depth description of this dataset.

We set $X_{k}^{\mu}=X_{k}^{\lambda}=\left(1, x_{k}, y_{k}\right)$, where $x_{k}$ and $y_{k}$ are respectively the latitude and the longitude at location $k, k=1, \ldots, 79$. The marginal parameters are $\beta^{\mu}=\left(\beta_{0}^{\mu}, \beta_{1}^{\mu}, \beta_{2}^{\mu}\right), \beta^{\lambda}=$ $\left(\beta_{0}^{\lambda}, \beta_{1}^{\lambda}, \beta_{2}^{\lambda}\right)$ and the shape is $\xi$; hence $\theta$ has 10 unknown parameters. The SpatialExtremes package is used in order to compute the maximum pairwise likelihood estimate (MPLE) $\tilde{\theta}^{\text {obs }}$, $H\left(\tilde{\theta}^{\text {obs }}\right)$ and $J\left(\tilde{\theta}^{\text {obs }}\right)$. The pairwise score function is approximated by finite difference methods (Monahan, 2011, Section 8.6). The prior for $\theta$ is uniform in the space $(0,1000) \times(-300,300) \times$ $(0,1000) \times(-\infty,+\infty)^{6} \times(0, \infty)$, under the condition that $\Sigma$ is a proper covariance matrix. For ABC-cs we use importance sampling with a multivariate $t$ distribution with 5 degrees of freedom, centred at $\tilde{\theta}^{\text {obs }}$ and with scale matrix $2.5 \mathrm{~V}\left(\tilde{\theta}^{\mathrm{obs}}\right)$ as importance function. We draw $1.1 \times 10^{6}$ values from the importance density and fix $\epsilon$ to $0.5 \%$ quantile of the observed distances, so we end up with 5500 values from the ABC-cs posterior.

The results are compared with the MPLE, with the pairwise posterior (5) and with the non calibrated pairwise posterior, the latter two approximated by $5 \times 10^{4}$ MCMC samples. The marginal posteriors for $\sigma_{1}^{2}, \sigma_{12}, \sigma_{2}^{2}, \beta^{\lambda}$ are shown in Figure 7 , whereas Table 1 reports some numerical summaries for all the parameters. The plots of the other marginal posteriors are reported in the Supplementary Material.

Table 1 confirms that Bayesian inference based on the non calibrated pairwise likelihood can be overly too precise. The calibrated pairwise and the ABC-cs posteriors for the marginal parameters appear to be quite different, especially for the dependence parameters (first row of Figure 7). For instance, while the ABC-cs 0.95 credible interval for $\sigma_{12}$ does not include zero, the contrary holds for MCMC calibrated pairwise credible interval; the latter suggests that 

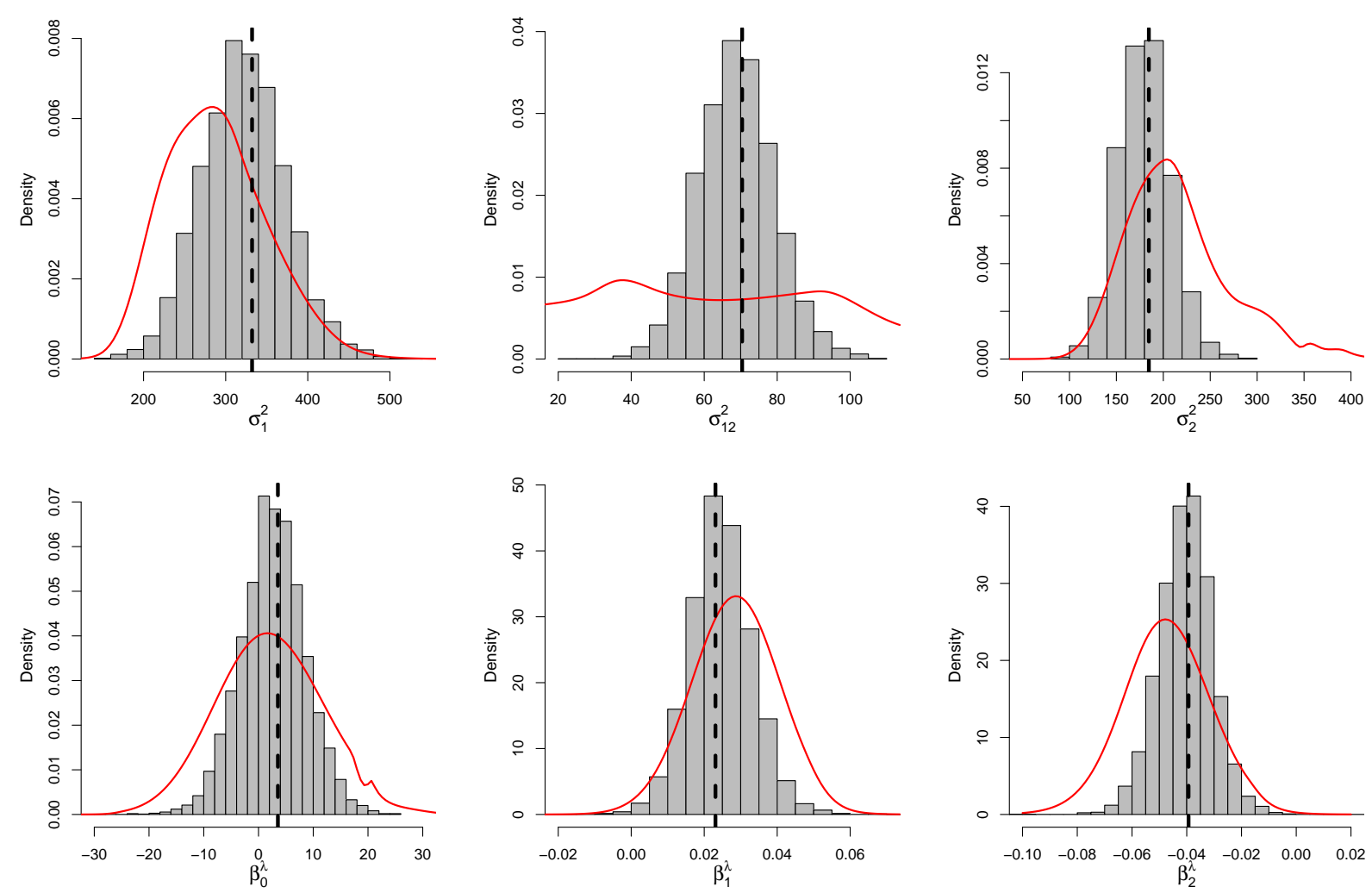

Figure 7: Swiss rainfall data. Marginal ABC-cs (histogram) and calibrated pairwise (kernel densities in red) posteriors compared with MPLE (dashed vertical lines). The first row shows the dependence parameters and the second row shows $\beta^{\lambda}$.

the process may be isotropic. The ABC-cs posterior is in good agreement with the MPLE and the corresponding standard errors, whereas the calibrated pairwise posterior appears to be too dispersed.

We further compare the MPLE with the ABC-cs and the calibrated pairwise posteriors by plotting the estimated pairwise extremal coefficient calculated at the corresponding means. These comparisons are shown on the left panel of Figure 8. Again we notice that ABC-cs is very similar to the MPLE, whereas the extremal coefficient based on the calibrated pairwise posterior appears substantially different. Moreover, this plot confirms that the calibrated pairwise posterior shows more isotropy than MPLE or ABC-cs. The right panel of Figure 8 shows the extremal coefficient as a function of the Euclidean distance among the locations, with 0.95 credible bands computed pointwise at each distance of $h$. This plot highlights 


\begin{tabular}{crrrr}
\hline Param. & MPLE (SE) & ABC-cs & Cal. pairwise & Pairwise \\
\hline$\sigma_{1}^{2}$ & $332.15(55.86)$ & $321.88(51.03)$ & $288.71(61.96)$ & $325.63(4.75)$ \\
$\sigma_{12}^{2}$ & $70.40(11.40)$ & $68.95(10.41)$ & $63.39(40.94)$ & $69.99(2.85)$ \\
$\sigma_{2}^{2}$ & $184.63(30.45)$ & $180.17(27.69)$ & $215.98(56.51)$ & $181.49(2.74)$ \\
$\beta_{0}^{\mu}$ & $20.65(8.67)$ & $21.09(9.09)$ & $22.62(12.22)$ & $22.79(0.563)$ \\
$\beta_{1}^{\mu}$ & $0.06(0.01)$ & $0.06(0.01)$ & $0.06(0.02)$ & $0.06(0.001)$ \\
$\beta_{2}^{\mu}$ & $-0.16(0.02)$ & $-0.15(0.02)$ & $-0.15(0.02)$ & $-0.15(0.001)$ \\
$\beta_{0}^{\lambda}$ & $3.54(5.56)$ & $2.65(5.97)$ & $2.01(9.62)$ & $2.35(0.43)$ \\
$\beta_{1}^{\lambda}$ & $0.02(0.01)$ & $0.02(0.01)$ & $0.03(0.01)$ & $0.03(0.001)$ \\
$\beta_{2}^{\lambda}$ & $-0.04(0.01)$ & $-0.04(0.01)$ & $-0.05(0.02)$ & $-0.05(0.001)$ \\
$\xi$ & $0.19(0.03)$ & $0.18(0.03)$ & $0.18(0.03)$ & $0.18(0.001)$ \\
\hline
\end{tabular}

Table 1: Swiss rainfall data. Means (and standard deviations) of ABC-cs, pairwise and calibrated pairwise posteriors, compared with the MPLE and its asymptotic standard error (SE).

that the extremal coefficients computed from the calibrated pairwise posterior show more variability than those obtained from ABC-cs. The Wald-type confidence bands of MPLE essentially overlap with those of ABC-cs and therefore are not reported.

\section{Discussion}

A new procedure for constructing summary statistics for ABC is proposed, which is based on a rescaled composite score function. An advantage of the proposed method is that, by construction, the summary statistics automatically incorporate relevant features of the complex model, and its dimension is the same as the number of parameters. Moreover, no post processing tasks are required, nor pilot runs or ad hoc summaries of the data. With a little additional computational effort, the Godambe information can be obtained as a byproduct of our method. Such information matrix can be used as a scaling matrix in simulation schemes. 

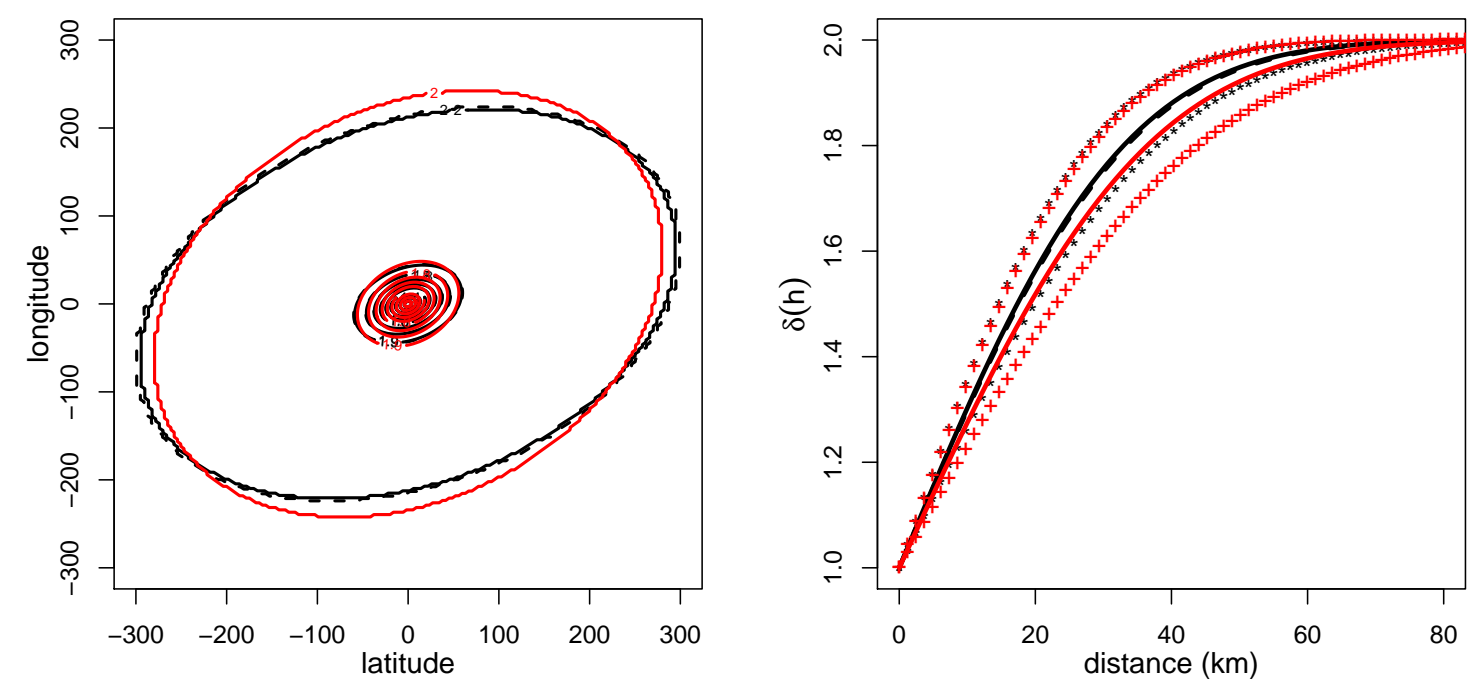

Figure 8: Left panel: pairwise extremal coefficient of the Smith model with the Swiss rainfall data computed using the ABC-cs posterior mean (continued line), the calibrated pairwise posterior mean (red line) and the MPLE (dashed). Right panel: pairwise extremal coefficient plotted as function of the Euclidean distance among the locations, with 0.95 pointwise credible bands from ABC-cs $(*)$ and calibrated pairwise posterior $(+)$.

Although being computationally more expensive than Bayesian composite posteriors, ABC-cs does not require calibration. Moreover, as seen from the examples and from the application, Bayesian inference with composite likelihoods, both calibrated and non calibrated, can be quite inaccurate.

The ABC-cs procedure depends of course on the availability of a reasonable composite likelihood for the problem under investigation, which may not be easy to obtain, or even define, in some problems. However, there is a rich and growing literature on composite likelihoods (Larribe \& Fearnhead, 2011; Varin et al., 2011), which we believe may be fruitfully used to identify the class of problems in which composite likelihoods may be used in ABC and also to guide the choice of the more appropriate composite likelihood. In general, a sensible composite likelihood has to be a good approximation for the full likelihood, or at least it has to appropriately describe the main features of interest of the model, by keeping a reasonable computational complexity. Even with this in mind, there could still be possible compet- 
ing composite likelihoods for the same model. Recent contributions in frequentist inference consider the idea of combining different composite likelihoods in order to improve efficiency (Cox \& Reid, 2004; Kenne Pagui et al., 2014). This idea could be used also in ABC-cs, and further extended to the combination of composite scores with other summary statistics.

Finally, we note that we used the composite likelihood as a natural basis to construct a suitable unbiased estimating function in complex models. However, the proposed ABC algorithm works with any unbiased estimating function, such as for instance those used in the robust literature (see, e.g., Huber \& Ronchetti, 2009).

\section{Supplementary Material}

The online Supplementary Material includes additional simulations and plots for the examples of Section 4 and the application in Section 5, and another example with a moving average process of order 2 . The $\mathrm{R}$ code for the examples and the application is also included.

\section{Acknowledgements}

We are grateful to two anonymous Reviewers for their thoughtful comments which lead to a substantially improved version of our initial draft. We thank Manuela Cattelan for sharing her code on the multivariate probit example. This work was supported by a grant from the University of Padua (Progetti di Ricerca di Ateneo 2013) and by the Cariparo Foundation Excellence-grant 2011/2012.

\section{References}

Barthelmé, S. \& Chopin, N. (2014). Expectation propagation for likelihood-free inference. Journal of the American Statistical Association 109, 315-333.

Beaumont, M. A. (2010). Approximate Bayesian computation in evolution and ecology. Annual Review of Ecology, Evolution, and Systematics 41, 379-406. 
Beaumont, M. A., Cornuet, J.-M., Marin, J.-M. \& Robert, C. P. (2009). Adaptive approximate Bayesian computation. Biometrika 96, 983-990.

Beaumont, M. A., Zhang, W. \& Balding, D. J. (2002). Approximate Bayesian computation in population genetics. Genetics 162, 2025-2035.

Blanchet, J. \& Davison, A. C. (2011). Spatial modeling of extreme snow depth. The Annals of Applied Statistics 5, 1699-1725.

Blum, M. G. B. (2010). Approximate bayesian computation: a nonparametric perspective. Journal of the American Statistical Association 105, 1178-1187.

Brazzale, A. R., Davison, A. C. \& Reid, N. (2007). Applied Asymptotics: Case Studies in Small-Sample Statistics. Cambridge: Cambridge University Press.

Buishand, T., De HaAn, L., Zhou, C. et al. (2008). On spatial extremes: With application to a rainfall problem. The Annals of Applied Statistics 27, 624-642.

Cattelan, M. \& Sartori, N. (2014). Empirical and simulated adjustments of composite likelihood ratio statistics. arXiv arXiv:1403.7093v1.

Chib, S. \& Greenberg, E. (1998). Analysis of multivariate probit models. Biometrika 85, 347-361.

Cox, D. R. \& ReID, N. (2004). A note on pseudolikelihood constructed from marginal densities. Biometrika 91, 729-737.

Davison, A. C. \& Gholamrezaee, M. M. (2012). Geostatistics of extremes. Proceedings of the Royal Society A: Mathematical, Physical and Engineering Science 468, 581-608.

Davison, A. C., Padoan, S. A. \& Ribatet, M. (2012). Statistical modeling of spatial extremes. Statistical Science 27, 161-186.

De HaAn, L. (1984). A spectral representation for max-stable processes. The Annals of Probability 12, 1194-1204. 
Del Moral, P., Doucet, A. \& Jasra, A. (2012). An adaptive sequential Monte Carlo method for approximate Bayesian computation. Statistics and Computing 22, 1009-1020.

Drovandi, C. C. \& Pettitt, A. N. (2011). Estimation of parameters for macroparasite population evolution using approximate Bayesian computation. Biometrics 67, 225-233.

Drovandi, C. C., Pettitt, A. N. \& Faddy, M. J. (2011). Approximate Bayesian computation using indirect inference. Journal of the Royal Statistical Society: Series C 60, 317-337.

Drovandi, C. C., Pettitt, A. N. \& Lee, A. (2014). Bayesian indirect inference using a parametric auxiliary model. Statistical Science, to appear .

Erhardt, R. J. \& Smith, R. L. (2012). Approximate Bayesian computing for spatial extremes. Computational Statistics \& Data Analysis 56, 1468-1481.

Fearnhead, P. \& Prangle, D. (2012). Constructing summary statistics for approximate Bayesian computation: semi-automatic approximate Bayesian computation (with Discussion). Journal of the Royal Statistical Society: Series B 74, 419-474.

Fraser, D. A. S. \& Reid, N. (1995). Ancillaries and third order significance. Utilitas Mathematica 47, 33-53.

Genton, M. G., Ma, Y. \& Sang, H. (2011). On the likelihood function of Gaussian max-stable processes. Biometrika 98, 481-488.

Gleim, A. \& Pigorsch, C. (2013). Approximate Bayesian computation with indirect summary statistics. Technical report, University of Bonn.

Gourieroux, C., Monfort, A. \& Renault, E. (1993). Indirect inference. Journal of Applied Econometrics 8, S85-S118.

Heggland, K. \& Frigessi, A. (2004). Estimating functions in indirect inference. Journal of the Royal Statistical Society: Series B 66, 447-462. 
Huber, P. J. \& Ronchetti, E. M. (2009). Robust Statistics. Hoboken, New Jersey: Wiley.

Huser, R. \& Davison, A. C. (2013). Composite likelihood estimation for the BrownResnick process. Biometrika 100, 511-518.

Jung, H. \& Marjoram, P. (2011). Choice of summary statistic weights in approximate Bayesian computation. Statistical Applications in Genetics and Molecular Biology 10, Article 45.

Kabluchko, Z., Schlather, M. \& De HaAn, L. (2009). Stationary max-stable fields associated to negative definite functions. The Annals of Probability 37, 2042-2065.

Kenne Pagui, E. C., Salvan, A. \& Sartori, N. (2014). Combined composite likelihood. The Canadian Journal of Statistics 42, 525-543.

Kenne Pagui, E. C., Salvan, A. \& Sartori, N. (2015). On full efficiency of the maximum composite likelihood estimator. Statistics \& Probability Letters 97, 120-124.

Larribe, F. \& Fearnhead, P. (2011). On composite likelihoods in statistical genetics. Statistica Sinica 21, 43-69.

Le Cessie, S. \& van Houwelingen, J. C. (1994). Logistic regression for correlated binary data. Journal of the Royal Statistical Society: Series C 43, 95-108.

Lindsay, B. G. (1988). Composite likelihood methods. Contemporary Mathematics 80, 220-239.

Lindsay, B. G., YI, G. Y. \& Sun, J. (2011). Issues and strategies in the selection of composite likelihoods. Statistica Sinica 21, 71-105.

Marin, J.-M., Pudlo, P., Robert, C. P. \& Ryder, R. J. (2012). Approximate Bayesian computational methods. Statistics and Computing 22, 1167-1180.

Marjoram, P., Molitor, J., Plagnol, V. \& Tavaré, S. (2003). Markov chain Monte Carlo without likelihoods. Proceedings of the National Academy of Sciences 100, 1532415328. 
Mengersen, K. L., Pudlo, P. \& Robert, C. P. (2013). Bayesian computation via empirical likelihood. Proceedings of the National Academy of Sciences 110, 1321-1326.

Molenberghs, G. \& Verbeke, G. (2005). Models for Discrete Longitudinal Data. New York: Springer.

Monahan, J. F. (2011). Numerical Methods of Statistics. Cambridge University Press, 2nd ed.

Pace, L. \& Salvan, A. (1997). Principles of Statistical Inference. Singapore: World Scientific.

Pace, L., Salvan, A. \& Sartori, N. (2011). Adjusting composite likelihood ratio statistics. Statistica Sinica 21, 129-148.

Padoan, S. A., Ribatet, M. \& Sisson, S. A. (2010). Likelihood-based inference for max-stable processes. Journal of the American Statistical Association 105, 263-277.

Pauli, F., Racugno, W. \& Ventura, L. (2011). Bayesian composite marginal likelihoods. Statistica Sinica 21, 149-164.

ReID, N. (2003). Asymptotics and the theory of inference. The Annals of Statistics 31, $1695-1731$.

Ribatet, M., Cooley, D. \& Davison, A. C. (2012). Bayesian inference from composite likelihoods, with an application to spatial extremes. Statistica Sinica 22, 813-845.

Ribatet, M., Singleton, R. \& team, R. C. (2013). SpatialExtremes: Modelling Spatial Extremes. R package version 2.0-0.

Sang, H. \& Genton, M. G. (2014). Tapered composite likelihood for spatial max-stable models. Spatial Statistics 8, 86-103.

Schlather, M. (2002). Models for stationary max-stable random fields. Extremes 5, 33-44. 
Sisson, S., FAn, Y. \& TAnaka, M. (2007). Sequential Monte Carlo without likelihoods. Proceedings of the National Academy of Sciences of the United States of America 104, 1760-1765.

Sisson, S., FAn, Y. \& TAnaka, M. (2009). Sequential Monte Carlo without likelihoods: Errata. Proceedings of the National Academy of Sciences of the United States of America 106, 16889.

Smith, E. L. \& Stephenson, A. G. (2009). An extended Gaussian max-stable process model for spatial extremes. Journal of Statistical Planning and Inference 139, 1266-1275.

Smith, R. L. (1990). Max-stable processes and spatial extremes. Unpublished manuscript, University of North Carolina.

Varin, C., Reid, N. \& Firth, D. (2011). An overview of composite likelihood methods. Statistica Sinica 21, 5-42. 